\title{
Fatores facilitadores e dificultadores reportados por filhas ao lidarem com a doença e o tratamento oncológico das mães
}

\author{
Mariana Gil \\ Manoel Antônio dos Santos
}

\begin{abstract}
RESUMO
0 câncer de mama é causa relevante de morbimortalidade feminina. Embora as repercussões na família sejam alvo de inúmeros estudos, os impactos psicológicos sobre as filhas permanecem pouco explorados. 0 objetivo deste estudo foi investigar os fatores que facilitam ou dificultam o enfrentamento de filhas de mulheres acometidas pelo câncer de mama na situação de doença e tratamento oncológico de suas mães. Participaram 11 filhas de mulheres que cuidaram de suas mães adoecidas. Foram realizadas entrevistas individuais, audiogravadas e transcritas na íntegra. Os relatos foram submetidos à análise temática indutiva. Foram identificados fatores facilitadores e dificultadores. Em relação aos primeiros, as filhas destacaram a fé inspirada por alguma força maior ou religião, o apoio recebido de familiares e a percepção da força e coragem materna na luta pela sobrevivência. Por outro lado, a falta de apoio por parte de alguns membros da família e dos serviços médicos e oncológicos fragilizou o processo de enfrentamento. Os resultados destacam a importância da oferta de apoio social e cuidado especializado às filhas cujas mães são acometidas por uma doença carregada de estigmas e preconceitos, e que ainda apresenta altas taxas de letalidade em países em desenvolvimento como o Brasil.
\end{abstract}

Palavras-chave: neoplasias da mama; filhas; cuidadores; enfrentamento; saúde da mulher

\section{ABSTRACT}

\section{Facilitating and hindering factors reported by daughters when dealing with mothers' disease and cancer treatment}

Breast cancer is a relevant cause of female morbidity and mortality. Although the repercussions on the family are the subject of numerous studies, the psychological impacts on the daughters remain little explored. This study aimed to investigate the factors that facilitate or hinder the coping of daughters of women affected by breast cancer in the situation of disease and cancer treatment of their mothers. Participants included eleven daughters of women who took care of their sick mothers. Individual interviews were recorded, audio-recorded and transcribed in full. The reports were submitted to inductive thematic analysis. Facilitating and hindering factors were identified. Considering the facilitating factors, the daughters highlighted the faith inspired by some superior strength or religion, the support received from family members and the perception of the courage and strength of the mother who fights for survival. On the other hand, the lack of support from some family members and medical and cancer services weakened the coping process. The results highlight the importance of offering social support and specialized care to daughters whose mothers are afflicted with a disease laden with stigma and prejudice, and which still has high lethality rates in developing countries like Brazil.

Keywords: breast neoplasms; daughters; caregivers; coping behavior; women's health

0 câncer de mama é o segundo tipo de câncer mais frequente no mundo e consiste em uma das maiores causas de morte em mulheres, ocorrendo mais frequentemente após os 35 anos de idade (Brasil, 2020; Cesnik \& Santos, 2012a, 2012b). Para 2020 estima-se que haverá 66.280 casos novos de câncer de mama, sendo o tipo de neoplasia com maior incidência em mulheres

\section{Sobre os autores}

M. G.

https://orcid.org/0000-00018170-885X

Faculdade de Filosofia, Ciências e Letras de Ribeirão Preto, Universidade de São Paulo, Ribeirão Preto - SP, Brasil. marigil561@gmail.com

\section{A. S.}

https://orcid.org/0000-0001-

8214-7767

Faculdade de Filosofia, Ciências e Letras de Ribeirão Preto, Universidade de São Paulo, Ribeirão Preto - SP, Brasil.

masantos@ffclrp.usp.br

\section{Direitos Autorais}

Este é um artigo de acesso aberto e pode ser reproduzido livremente, distribuído, transmitido ou modificado, por qualquer pessoa desde que usado sem fins comerciais. 0 trabalho é disponibilizado sob a licença Creative Commons CC-BY-NC.

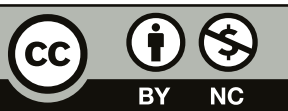




\section{M." INTERACÃO EM 2. PSICOLOGIA}

no Brasil, representando 29,7\% dos casos de câncer. Em 2017 foi o tipo de câncer que mais matou mulheres, representando $16 \%$ do total de óbitos femininos por neoplasia (Brasil, 2020). 0 crescimento contínuo da incidência das neoplasias mamárias representa um sério problema de saúde pública (Andolhe et al., 2009; Ferreira et al., 2015; Fonseca et al., 2014).

Por sua elevada incidência e também por proporcionar elevadas taxas de sobrevivência na atualidade, o câncer de mama apresenta intensas repercussões físicas e psicológicas em muitas mulheres, mesmo após um longo tempo decorrido desde a descoberta e o tratamento (Cesnik et al., 2013; Emslie et al., 2007; Inocenti et al., 2016; Junqueira et al., 2013; Santos \& Souza, 2019; Silva et al., 2005; Vidotti et al., 2013; Vieira et al., 2013, 2014). Estudo com 16 mulheres submetidas à mastectomia (retirada da mama) total ou parcial, seguida de quimioterapia e/ou radioterapia, identificou estressores físicos e sequelas duradouras que não se limitam ao corpo e organismo das pacientes (Silva \& Santos, 2008). As participantes relataram ter vivenciado dificuldades familiares e problemas sexuais decorrentes da quimioterapia e radioterapia, dores e redução da amplitude de movimento do braço homolateral à cirurgia, mobilidade reduzida e hipertensão arterial.

Além dos diversos agravos físicos decorrentes do tratamento da neoplasia mamária, a literatura reporta que as repercussões familiares e sociais são tão ou mais importantes na depreciação da qualidade de vida da maioria das mulheres que enfrentam o câncer de mama. Em relação aos efeitos psicológicos, Schmid-Büchi et al. (2013) investigaram as principais necessidades de apoio de 177 mulheres recém-diagnosticadas com câncer de mama, e os resultados indicaram que as questões psicológicas se destacaram como aquelas que as pacientes consideravam que mais necessitavam receber ajuda nessa etapa do tratamento. Os fatores psicológicos de vulnerabilidade mais relevantes para as mulheres consistiam em dificuldades no relacionamento interpessoal e alterações na imagem corporal.

Ao considerar os diferentes tipos de repercussões e limitações que o câncer de mama e seu tratamento podem acarretar na vida da mulher acometida, compreende-se que, ao atravessar essa situação-limite, ela terá necessidade acentuada de receber suporte familiar (Santos et al., 2014a, 2014b, 2017). De todas as possíveis fontes de apoio, aquela que é considerada a mais importante nesse momento da vida da mulher é o suporte familiar (Ambrósio \& Santos, 2011; Hoffmann et al., 2006; Souza et al., 2014). Nos estudos de Hoffmann et al. (2006) e Yoshimochi et al. (2018), a principal fonte de apoio referida pelas entrevistadas eram parentes próximos, companheiros e amigos, sendo que os primeiros foram mencionados por todas as mulheres como importante recurso de suporte social. A cirurgia a que a mulher é submetida pode ser conservadora ou radical. Silva e Santos (2008) apontam que, após a intervenção cirúrgica, a mulher pode vivenciar conflitos relacionados aos significados atribuídos à mama. Nesse momento crítico, o suporte dos filhos e do companheiro torna-se de extrema relevância, no sentido de auxiliar a mulher a aceitar sua condição de mastectomizada, verbalizar seus traumas e sentimentos, e tornar-se participativa no autocuidado e na prevenção de novos agravos.

Por outro lado, é preciso considerar que o sistema familiar também sofre os impactos do adoecer e do tratamento oncológico (Ambrósio \& Santos, 2015; Silva et al., 2019; Yoshimochi et al., 2018). Ou seja, as repercussões do câncer de mama não incidem apenas sobre a mulher acometida, mas se ramificam e se estendem também a seus familiares (Biffi \& Mamede, 2010). A estrutura familiar é abalada pelo câncer de mama, que tem sua maior incidência a partir da quarta década de vida, quando a mulher está exercendo múltiplos papéis familiares e sociais que são vitais para a reprodução da vida familiar. Estudo mostrou que há uma fina sintonia entre a condição emocional da mãe e dos demais membros da família. Se a mulher acometida pelo câncer se encontra em determinado estado de ânimo, o restante da família também tende a ser afetado pelo mesmo estado, e vice-versa. As mudanças adaptativas que a doença exige dos familiares são mobilizadoras de estresse, o que acaba afetando o estado de humor, de modo que os filhos também podem ser, de algum modo, afetados tanto em sua rotina quanto no seu funcionamento psicológico, o que deflagra uma situação de vulnerabilidade (Sanchez et al., 2010).

Logo após a revelação do diagnóstico de câncer de mama, os familiares, em um primeiro momento, referem sentir desespero e medo do que pode acontecer no futuro, e muitas vezes significam o diagnóstico como uma sentença antecipadora da morte de seu ente querido. Diante das situações extraordinárias evocadas pelo câncer de mama e vivenciadas de forma difusa por todo o sistema familiar, alguns membros da família referem ter sido levados a pensar mais seriamente sobre fatores existenciais, tais como a finitude e o sentido da vida. Mesmo após a conclusão do tratamento, são identificados nos familiares sintomas de ansiedade persistente e tensão latente relacionada ao temor da recidiva, à ameaça de eclodir mais um caso de câncer na família e também ao prenúncio de morte, iminente ou não, despertado pelo câncer (Ambrósio \& Santos, 2017; Araújo \& Nascimento, 2004; Tavares \& Trad, 2005).

No estudo de Sales et al. (2001), o componente familiar mais reconhecido como fonte de apoio pelas pacientes foram os filhos, sendo que $77 \%$ das mulheres relataram que conversavam sobre o assunto com eles. A dependência de cuidados se intensifica nas diversas etapas do tratamento e de acordo com a evolução da doença oncológica. Geralmente, a divisão social dos papéis familiares segundo o gênero deposita nas filhas a função de cuidadoras principais de suas mães adoeci- 


\section{MLE INTERACÃO EM PSICOLOGIA}

das. No entanto, embora se perceba certa preocupação na literatura em investigar as repercussões psicológicas do câncer de mama nos companheiros e demais familiares das mulheres acometidas pela doença, pouco se sabe sobre o impacto psicológico específico nas filhas e filhos dessas mulheres, e menos ainda sobre as repercussões nas filhas que ampararam suas mães diretamente, ao longo do processo de adoecimento.

No que concerne especificamente às filhas de mulheres acometidas pelo câncer de mama, pesquisa de Raveis e Pretter (2005) revelou que elas se percebem em uma situação desafiadora, ao terem de lidar com a sobrecarga de tarefas inerentes ao cuidado. Muitas vezes as filhas sentem que as demandas excedem os recursos de que dispõem para lidar com as dificuldades que aparecem na longa jornada do tratamento oncológico. Elas referem que se veem confrontadas com a necessidade de oferecerem continência e acolherem as reações psicológicas de suas mães, ao mesmo tempo em que têm de lidar com suas próprias emoções, que tendem a ser exacerbadas no decorrer do percurso. As filhas tornam-se mais suscetíveis a sentimentos contraditórios, mobilizados enquanto exercem o papel de cuidadoras, acompanhando suas mães nas consultas médicas, exames, retornos e eventuais internações. As filhas também relatam que procuram alternativas que poderiam auxiliá-las a amenizar suas inquietações e a mitigar seu sofrimento, mas que não se sentem à vontade para conversarem abertamente com suas mães sobre essas preocupações. Mencionam que não se sentem confortáveis para expor o que pensam e sentem, temendo que essa transparência na comunicação possa agravar a fragilidade materna.

Araújo e Nascimento (2004) entrevistaram dez familiares de mulheres com câncer de mama. Apenas uma das participantes era filha. Essa colaboradora relatou que, diante da presença da doença em sua vida familiar, passou a se preocupar com o fato de também poder desenvolver a doença, em vista de seu componente genético, além de ter passado a se preocupar com formas de prevenção da neoplasia mamária. Outro estudo confirmou que filhas cujas mães vivenciaram o câncer de mama apresentam preocupações em relação ao futuro com frequência significativamente maior do que filhas de mulheres saudáveis (Cappelli et al., 2005).

Enfrentamento pode ser definido como um processo pelo qual um indivíduo maneja o estímulo que julga ser estressante (Lazarus \& Folkman, 1984). Cada pessoa utiliza estratégias de enfrentamento que, de acordo com sua avaliação, poderão ser mais efetivas para superar o estresse resultante da exposição ao evento estressor (Andolhe et al., 2009; Arruda-Colli et al., 2018). No cenário do câncer de mama existem estressores específicos que precisam ser considerados no processo de enfrentamento familiar (Compas et al., 1996; Silva \& Santos, 2010; Yates, 1999). No caso das filhas das mulheres que de- senvolveram o câncer de mama, tais estratégias configuram um conjunto de esforços desenvolvidos para administrar os estímulos internos e externos. Nessa acepção, o enfrentamento é a resposta que as filhas dão aos diferentes estímulos, a fim de manterem sua integridade física e psíquica, na tentativa de neutralizar e/ou eliminar os estressores relacionados ao cuidado das mães adoecidas e, assim, restabelecer seu equilíbrio interno (Davey et al., 2005). O enfrentamento focalizado na emoção é definido pelos esforços mobilizados para regular o estado emocional e pelas emoções associadas quando a situação é entendida como inalterável, ao passo que no enfrentamento focado no problema a situação é avaliada como passível de ser alterada pela eliminação ou modificação das circunstâncias que geram o problema (Lazarus \& Folkman, 1984).

O modo como os filhos adolescentes significam e lidam com situações adversas influencia sua capacidade de ajustamento psicológico à experiência do adoecimento materno (Compas et al., 1996; Yates, 1999). Todavia, os modos de enfrentamento dos adolescentes e de outros membros da família utilizados durante o processo de diagnóstico e tratamento ainda não são bem conhecidos (Ambrósio \& Santos, 2011; Compas et al., 1996). Segundo a literatura, a maioria das crianças e adolescentes mostra ter uma avaliação cognitiva precisa do câncer de seus pais, todavia, do ponto de vista emocional, o impacto parece ser potencialmente disruptivo. Se as adolescentes percebem a doença como ameaçadora à continuidade da vida das mães, têm maior probabilidade de usar estratégias de enfrentamento potencialmente não adaptativas, ou seja, focalizadas na emoção, e não no problema, com o objetivo de manterem as consequências emocionais da situação adversa dentro de limites aceitáveis (Compas et al., 1996; Davey et al., 2005).

Para compreender as potenciais repercussões do câncer materno sobre as filhas, é necessário considerar que a doença tem claramente um componente genético. Logo, a presença de antecedentes familiares pode levar os descendentes, especialmente as filhas, a se preocuparem com a possibilidade de terem uma predisposição aumentada para desenvolverem a mesma neoplasia que acometeu suas mães. Isso ficou incrementado após o episódio, fartamente explorado pela mídia, da atriz Angelina Jolie, que mesmo estando saudável decidiu se submeter à mastectomia bilateral radical como medida profilática. Assim, ao compreender os impactos psicossociais que incidem sobre os familiares da mulher com câncer de mama, é legítimo esperar que as filhas possam vivenciar sentimentos que vão além das preocupações normalmente vivenciadas pelos demais familiares - marido e filhos. Todavia, a literatura dedicada a essa temática é escassa e raramente focaliza a investigação das estratégias de enfrentamento mobilizadas pelas filhas face à situação de doença e tratamento de suas mães, o que justifica a proposta da presente pesquisa. 


\section{H MTERAC̄OOEM E. PSICOLOGIA}

Este estudo teve por objetivo investigar os fatores que facilitam ou dificultam o enfrentamento de filhas de mulheres acometidas pelo câncer de mama na situação de doença e tratamento oncológico de suas mães.

\section{MÉTODO}

Trata-se de um estudo descritivo-exploratório, de corte transversal e abordagem qualitativa.

\section{PARTICIPANTES}

Participaram deste estudo 11 filhas de mulheres acometidas por câncer de mama, vinculadas a um centro de reabilitação de mulheres mastectomizadas de uma cidade do interior do Estado de São Paulo. As participantes tinham entre 18 e 66 anos (média de idade igual a 32 anos), sete eram casadas, e quatro, solteiras, seis delas tinham filhos (variando de um a três).

As participantes foram selecionadas por atenderem aos seguintes critérios de inclusão: ser filha de mulher diagnosticada com câncer de mama, independentemente da idade, grau de estadiamento da doença e tempo de diagnóstico; ter idade igual ou acima de 18 anos; ter sido cuidadora, definida como aquela pessoa da família que participou ativamente e ofereceu apoio durante o período de diagnóstico e tratamento da mãe, acompanhando-a durante as consultas médicas, realização de exames, hospitalização, ciclos de quimioterapia e sessões de radioterapia; e ter disponibilidade para participar voluntariamente da pesquisa. Foram critérios de exclusão: filhas cujas mães haviam falecido; dificuldades cognitivas ou de comunicação, que poderiam inviabilizar a aplicação da entrevista. A interrupção da coleta de dados foi determinada por saturação.

\section{INSTRUMENTOS}

Para a coleta de dados foram utilizados os seguintes instrumentos:

(1) Formulário de Dados Sociodemográficos: consiste em um questionário que permitiu identificar as participantes em termos de idade, estado civil, nível de escolaridade, entre outros dados, complementados pelos itens de conforto doméstico e grau de escolaridade do chefe da família (Critério de Classificação Econômica Brasil).

(2) Roteiro de Entrevista Semiestruturada: desenvolvido pelos pesquisadores, a partir de uma revisão de estudos sobre a temática e da experiência de mais de duas décadas do grupo de acompanhamento clínico e pesquisa especificamente com a população de mulheres mastectomizadas.
(3) Diário de campo: que consistiu nas anotações realizadas pela pesquisadora ao longo das entrevistas realizadas, incluindo suas impressões, sentimentos, inquietações e pensamentos decorrentes da experiência vivenciada durante a coleta de dados e logo depois dela.

\section{PROCEDIMENTO}

Coleta de dados. A coleta de dados foi realizada individualmente, em situação face a face, com as participantes que preenchiam os critérios de elegibilidade para o estudo. A entrevista foi realizada ao longo de um único encontro, no local de preferência das participantes. Nove escolheram conceder a entrevista em uma sala reservada do serviço de reabilitação ao qual as mães estavam vinculadas, e duas preferiram receber a pesquisadora em suas casas. As entrevistas tiveram duração média de 48,2 minutos. Foram audiogravadas em gravador digital, mediante anuência das participantes, e transcritas literalmente e na íntegra para posterior análise. Os dados do diário de campo foram redigidos logo após o encerramento da entrevista, de modo a assegurar uma descrição o mais completa possível da experiência que acabara de ocorrer.

Análise de dados. As entrevistas foram transcritas na íntegra e literalmente. O material coligido constituiu o corpus da pesquisa. Posteriormente, os dados foram organizados e submetidos à análise temática, segundo os passos preconizados por Braun e Clarke (2006), visando a identificar concepções, crenças, valores, motivações, expectativas e atitudes comuns entre os participantes do estudo. Segundo essas autoras, a análise temática indutiva é bastante útil e flexível para a pesquisa qualitativa em Psicologia. É um método que permite identificar, analisar e relatar padrões (temas) nos dados obtidos, de forma que o pesquisador tem um papel ativo ao longo desse processo. A análise temática é minimamente organizada e possibilita descrever o conjunto de dados em detalhes, em um processo orientado e dirigido pelo interesse teórico do pesquisador.

Primeiramente, foram realizadas sucessivas e cuidadosas leituras do material transcrito, que proporcionou a sistematização preliminar das ideias-chave que emergiram em cada entrevista, a partir das quais se organizaram os eixos temáticos encontrados. 0 próximo passo foi realizar uma análise horizontal de todas as entrevistas realizadas, identificando os pontos de semelhança e também as diferenças observadas nos relatos do conjunto de participantes, a partir dos eixos temáticos encontrados. Por fim, os dados obtidos foram discutidos com base nos achados da literatura e interpretados à luz da teoria do enfrentamento. 


\section{4.' INTERACÃO EM ET. PSICOLOGIA}

\section{CONSIDERAÇÕES ÉTICAS}

Após obter a autorização da coordenadora do serviço de reabilitação de mastectomizadas, o projeto foi aprovado pelo Comitê de Ética em Pesquisa da Faculdade de Filosofia, Ciências e Letras de Ribeirão Preto, Universidade de São Paulo ( $\mathrm{Pa}$ recer: 890.730), resguardando-se os procedimentos éticos de respeito às voluntárias e à instituição, de acordo com a Resolução 466/2012 sobre Pesquisa Envolvendo Seres Humanos, do Conselho Nacional de Saúde (Brasil, 2012). Em atendimento às diretrizes da Resolução, o projeto também foi encaminhado para apreciação do Comitê de Ética em Pesquisa da instituição coparticipante (Escola de Enfermagem de Ribeirão Preto - EERP), tendo sido aprovado.

Foi condição imprescindível para a abordagem das potenciais participantes que a mãe (paciente com história de câncer de mama) concordasse antecipadamente com a abordagem da filha. Caso estivessem de acordo, era então solicitado que conversassem com as filhas e verificassem seu interesse em colaborar com o desenvolvimento da pesquisa. Quando o retorno era positivo, a pesquisadora entrava em contato com a potencial participante e agendava a entrevista.

As participantes foram antecipadamente esclarecidas acerca dos objetivos do estudo, assim como das condições de resguardo do sigilo em relação aos dados fornecidos. Para formalizar sua anuência com a pesquisa, todas firmaram o Termo de Consentimento Livre e Esclarecido (TCLE).

\section{RESULTADOS E DISCUSSÃO}

Considerando o objetivo do estudo, a sistematização dos resultados permitiu identificar duas categorias temáticas - $f a-$ tores facilitadores e fatores dificultadores - que agrupam os sentidos atribuídos pelas filhas ao seu processo de lidar com as repercussões da doença e do tratamento oncológico de suas mães. Essas categorias abrigam algumas subcategorias que possibilitaram analisar, de forma compreensiva, o tema estudado e apreender com maior clareza como os resultados obtidos respondem à questão de pesquisa.

Fatores facilitadores são definidos, no contexto deste estudo, como quaisquer elementos que estimulam, proporcionam ou promovem um ambiente que facilita o enfrentamento da situação de adoecimento e tratamento. Inversamente, fatores dificultadores correspondem aos aspectos identificados pelas filhas como barreiras que encontraram ao lidarem com o processo de adoecer e o tratamento oncológico de suas mães. As duas categorias e suas respectivas subcategorias encontram-se sistematizadas na sequência.

\section{FATORES FACILITADORES AO LIDAR COM A DOENÇA E 0 TRA-} TAMENTO DA MÃE

Em relação aos fatores facilitadores do processo de lidar com os desafios colocados pela doença e tratamento da mãe, foram mencionadas circunstâncias que tornaram o enfrentamento mais fácil e menos doloroso por parte das filhas. Percebeu-se que essa categoria obteve elevada convergência entre diferentes relatos das participantes.

Essa categoria foi constituída pelas falas que apontaram para a centralidade do apoio familiar, do significado da espiritualidade/religiosidade como um recurso potente para lidar com as angústias e a percepção da força e destemor da mãe. As subcategorias encontradas foram:

- Apoio da família (presente nas falas de nove participantes);

- A fé e/ou religião (esta subcategoria emergiu nas falas de oito participantes);

- A coragem e força da mãe (esta subcategoria emergiu nos relatos de sete participantes).

0 primeiro fator identificado como facilitador do processo de lidar com a doença da mãe foi o apoio da família. Algumas filhas relataram ter recebido a colaboração de forma ampla, como um apoio por parte de familiares em geral, sem apontar um específico, ao passo que outras detalharam quais foram os familiares que efetivamente as apoiaram nos cuidados prestados à mãe, por conseguinte favorecendo seu próprio enfrentamento da situação. 0 excerto de fala a seguir ilustra essa subcategoria.

Então, a minha irmã está me ajudando demais, ela fica presente o mais que ela pode, ela só não fica quando não tem mesmo jeito... E tem uma outra irmã também que está me dando muito apoio, os meus irmãos estão me dando força. Todos eles. Graças a Deus estou contando com isso. Isso ajuda bastante. Eu acho que, se não fosse isso, eu desabava, né? Eles estão sempre juntos, me ligando: "E aí, como que está?", "Se precisar de mim, você me liga". Outra hora está junto, assim. Então, estão muito presentes, minha família está muito presente, graças a Deus (Elis, 66 anos).

Estudos apontam que a família é a fonte de apoio mais reconhecida pelas mulheres acometidas pelo câncer de mama (Ambrósio \& Santos, 2017; Hoffmann et al., 2006; Yoshimochi et al., 2018), mas o sistema familiar também sofre o impacto da doença, ou seja, as repercussões do câncer de mama se estendem também aos membros da família e afetam sua dinâmica de funcionamento (Biffı \& Mamede, 2010). Assim, é esperado que os familiares se apoiem mutuamente, uma vez que todos são suscetíveis de alguma forma aos efeitos do acometimento da mulher. Estudo quantitativo realizado com 


\section{M." INTERACÃO EM PSICOLOGIA}

147 filhas de mulheres com neoplasia mamária mostrou que cabia a elas prover apoio à mãe, mas elas também recebiam apoio de outros membros da família e de terceiros. Essas cuidadoras apresentavam menos sintomas de depressão do que as outras filhas, o que demonstra que o apoio (tanto o provido quanto o recebido) pode facilitar o processo de enfrentamento da filha em relação aos desafios da doença materna (Vodermaier \& Stanton, 2012). Os resultados do presente estudo são congruentes com os achados de outras pesquisas, que evidenciam como o apoio de outras pessoas se torna vital nesse momento desafiador.

A fé, enquanto dimensão constitutiva da espiritualidade, ou mesmo a religiosidade, que é a manifestação da espiritualidade em sua faceta institucionalizada (Benites et al., 2017; Borges et al., 2006; Cardoso et al., 2010; Freitas et al. 2017), configuraram uma subcategoria que esteve presente nos relatos de oito participantes. As filhas contam que o apoio da religião, a crença na existência de um Poder Superior e a fé e esperança na cura da mãe foram fontes determinantes de conforto e apoio para elas. Esses recursos favoreceram o enfrentamento de situações difíceis pelas quais a mãe passou, como exemplificado pela fala de uma participante:

... sou católica. E isso me ajudou muito. Na época, assim, era Deus e minha mãe, Deus e minha mãe, Deus e minha mãe (Ivete, 20 anos).

Estudos mostram que a crença e filiação religiosa, assim como a fé em Deus ou em alguma força maior constituem importantes alternativas de enfrentamento adotadas pelas muIheres acometidas por câncer de mama (Hoffmann et al., 2006; Peres \& Santos, 2009, 2010; Scorsolini-Comin et al., 2009; Tonon et al., 2016). O presente estudo evidencia que existe um processo semelhante nas filhas saudáveis, que buscam na relação com o sagrado na dimensão do sobrenatural um alento para mitigar o contato com situações de sofrimento, mobilizadoras de intensas angústias, que se não forem acolhidas podem levar a vivências de desamparo e devastação emocional. A literatura mostra que os familiares das pacientes também acreditam que a fé e o senso religioso podem levar à cura do câncer de mama. Eles também apoiam a crença da mulher em uma Força Superior que comanda a harmonia do universo, um Criador misericordioso que personifica a bondade, o que faz com que também acabem se utilizando dessa fonte como recurso para se fortalecerem frente aos seus próprios sentimentos de desamparo (Araújo \& Nascimento, 2004; Benites et al., 2021).

Outro fator facilitador do enfrentamento da doença oncológica materna, mencionado por sete participantes, foi a percepção de coragem e da força psicológica da mãe. As filhas se encontram em uma situação de tensionamento devido às inúmeras repercussões físicas, emocionais e sociais que o cân- cer desencadeia. Colocadas face ao imponderável e à possibilidade iminente da vida da mãe cessar em um futuro não muito distante, o fato de perceberem a mãe forte, com coragem para encarar as situações adversas com uma postura de aceitação, destemor e positividade auxiliou as filhas a enfrentarem com esperança os desdobramentos do câncer materno.

Eu percebi que: “Opa, pera aí, né, ela está forte, eu vou ficar assim, abalada?". . . É, foi mais tranquilo, eu acho que eu fiquei mais calma. Pensei que eu ia ficar tipo: "Nossa!", mas ela reagiu bem ao tratamento, foi tranquilo (Rafaela, 31 anos)

Ah, essa força dela, né. Porque ela tem mais força do que eu, nesse ponto. Ela é mais forte que eu. Eu falo: "Está doendo o joelho, mas nós temos que ir, mãe". "Ah, tá bom, então vamos". . . Aí, isso daí é o que está me dando mais força, né? É o que me dá mais força (Elis, 66 anos).

Vendo desde a perspectiva da paciente, Loyola e Muñíz (2013) argumentam que a mulher acometida pelo câncer de mama sente que deve apoiar sua família para que ela não desmorone emocionalmente diante da adversidade. Para tanto, muitas vezes a paciente se empenha em demonstrar uma força que não é real, mas aparente, preferindo ocultar dos familiares seus sentimentos genuínos, uma estratégia para poupá-los do contato com mais sofrimento. Não é possível afırmar se esse é o caso das sete mulheres cujas filhas relataram encontrar apoio na determinação e espírito de luta que perceberam em suas mães, mesmo porque as próprias filhas, provavelmente, não poderiam identificar se suas genitoras estariam aparentando ter uma força apenas para ampará-las, evitando sobrecarregá-las com mais inquietações e preocupações. De todo modo, é perceptível nos relatos das participantes que essa demonstração de coragem e estoicismo das mães facilitou o enfrentamento das próprias filhas. Isso é consistente com a literatura, que sugere que algumas mulheres manifestam uma reação emocional frente ao câncer de mama regulada pela crença de que devem suportar heroicamente o sofrimento; esse mecanismo de defesa é reconhecido na literatura da psico-oncologia como estoicismo (Peres \& Santos, 2007). Complementando esse achado, estudos mostram que o estado de humor do ente familiar acometido por câncer influencia diretamente o funcionamento emocional dos demais familiares, cujo equilíbrio emocional passa a acompanhar as oscilações de ânimo da pessoa adoecida, alternando frequentemente sentimentos de tristeza e alegria (Sales et al., 2010; Sanchez et al., 2010).

\section{FATORES DIFICULTADORES AO LIDAR COM A DOENÇA E 0 TRATAMENTO DA MÃE}

Essa categoria foi constituída pelas falas que apontam para a falta de apoio por parte de alguns familiares ou dos 


\section{M." INTERACÃO EM IT PSICOLOGIA}

serviços de saúde, levando à sobrecarga da filha cuidadora e aguçando a percepção da fragilidade da condição materna. As subcategorias encontradas foram:

- A saúde debilitada e o estado frágil da mãe, potencializando incertezas das filhas quanto à sua capacidade de superar a doença e se restabelecer (esta subcategoria apareceu na entrevista de cinco participantes).

- Falta de apoio por parte de alguns membros da família (esta subcategoria emergiu nas falas de quatro participantes).

- Falta de apoio por parte dos serviços especializados (três participantes).

A segunda categoria encontrada diz respeito aos fatores dificultadores identificados ao lidar com a doença e o tratamento da mãe, ou seja, circunstâncias e eventos que fizeram com que enfrentar a doença da mãe se tornasse mais difícil para as filhas cuidadoras. Um dos aspectos mais reiterados nessa categoria foi a saúde materna debilitada, que gera dúvidas na convicção da filha em relação à real possibilidade de a mãe vencer o câncer. Essas filhas relataram que ver suas mães debilitadas e se queixando frequentemente de dores e desconfortos físicos que nunca tinham presenciado anteriormente fez como que enfrentar o câncer de mama se tornasse mais difícil e penoso para elas. Além disso, também surgiram incertezas e temores acerca do futuro, pois já não sabiam se a mãe teria sequelas permanentes decorrentes dos tratamentos invasivos e, principalmente, temiam uma possível recidiva.

Eu não esperava essa... eu não esperava esse impacto tão grande durante a quimio, não esperava. Porque a gente vê a pessoa fazer a quimio sem cabelo... mas a gente só vê essa parte mais estética. Eu não imaginava que derrubava tanto a pessoa. Nunca imaginei que a pessoa ficasse tão mal (Gabriela, 37 anos).

Estudos mostram que o familiar que cuida de um parente acometido pelo câncer sofre ao presenciar os efeitos adversos do tratamento, e que também compartilha com o ente querido as dúvidas e incertezas que acompanham esse período crítico de suas vidas, justificando assim o porquê de esse ter sido um fator que dificultou o enfrentamento das filhas (Sales et al., 2010; Stiffler et al., 2008). Ainda segundo a literatura, é comum que filhas de mulheres com câncer de mama se deem conta pela primeira vez da vulnerabilidade de suas mães e passem a vê-las como mortais e frágeis, em sua condição humana, o que as obriga a se confrontarem com a ideia da inexorabilidade da finitude e com as incertezas que essa percepção reavivada causa (Raveis \& Pretter, 2005; Stiffler et al., 2008). Os resultados do presente estudo corroboram esses achados.

Outra subcategoria que emergiu foi a percepção da falta de apoio efetivo por parte de alguns membros da família. Apesar de três das quatro filhas também terem mencionado o apoio da família como fator facilitador do enfrentamento, elas apon- taram a falta de apoio de alguns familiares, como irmãos ou o pai, denotando a ausência ou incipiência de suporte como um fator dificultador do enfrentamento da situação. Essas participantes afirmaram que, se tivessem o apoio desses membros, seria mais fácil lidar com os problemas.

Ah, meus irmãos são muito ausentes, né? Que nem, quando aconteceu isso tudo com a minha mãe, eu precisava de aju$\mathrm{da}$, até certo ponto a gente precisa de ajuda. Não pra fazer as coisas pra ela, porque eu também não ia deixar que ninguém fizesse, porque eu ia querer estar junto. Mas, assim, tipo: podiam ir um pouco mais lá em casa, conversar um pouco mais com ela... Eles ficaram muito longe, muito distantes. Tipo, falaram: "Ah, a [nome da participante] está lá, então a gente não precisa se preocupar". Sempre foi isso, porque eu, por ser a mais nova, sempre fui a mais responsável e a mais cabeça da casa, então eles sempre... Eu que dava conselho, eu que chamava a atenção, eu que estava sempre tomando à frente de tudo, né? Então, acho que eles confiavam tanto em mim, que eles achavam que eu não precisava deles. [Pausa] Mas não sou eu, né. É a mãe, né? Eles precisavam ter dado um pouco mais de atenção pra minha mãe. E ela sente isso (Vanessa, 38 anos).

. . . meus pais e meus irmãos. Assim, cada um teve uma forma de expressar o que estava sentindo naquele momento. A minha forma de expressar foi parar minha vida e cuidar da minha mãe. A forma deles foi se afastar. Assim, eles conversavam, ficavam dentro de casa, mas nenhum deles conseguiu acompanhar ela numa quimioterapia, acompanhar ela numa radioterapia. Eles simplesmente não conseguiram (Ivete, 20 anos).

As participantes significam a falta de apoio de alguns membros da família como circunstância que afetou o bem-estar da mãe, deixando-a triste e desalentada. Esse foi um fator dificultador de seu próprio enfrentamento, deixando-as mais sensíveis e vulneráveis. Os estudos sugerem que o apoio familiar desde muito cedo torna-se um fator primordial, inclusive em mulheres que estão vivenciando a possibilidade de virem a ser mastectomizadas (Ambrósio \& Santos, 2015, 2017). Portanto, ausência ou apoio insuficiente podem acarretar prejuízos no processo adaptativo (Silva \& Santos, 2010).

A literatura aponta consistentemente que o que afeta a parente acometida por câncer também atinge o familiar que está cuidando dela, o que conflui para um fenômeno que se observou em algumas participantes deste estudo. Ao verem a mãe sofrendo pelo distanciamento de filhos e/ou do marido nesse momento delicado, acabaram também tendo seu enfrentamento dificultado por essa razão (Sales et al., 2010; Sanchez et al., 2010). Além disso, estudo realizado por Miranda e Aravena (2012) mostrou que a maior parte das cuidadoras assume a responsabilidade integral de cuidar do familiar acometido por alguma doença; na maioria das vezes elas cumprem as tarefas sozinhas, recebendo pouco apoio social, inclusive da 


\section{M." INTERACÃO EM 2. PSICOLOGIA}

própria família. Especialmente nos períodos mais críticos do tratamento (cirurgia, quimioterapia, radioterapia), a ausência ou insuficiência de suporte familiar aumenta a sobrecarga dos cuidadores (Ambrósio \& Santos, 2015; Bergerot et al., 2014; Inocenti et al., 2016).

É importante destacar a questão de gênero que atravessa esse processo de adjudicação do papel de cuidadora a uma única familiar. Estudos mostram que o cuidado da saúde física e mental da família recai quase sempre sobre as mulheres e que elas constituem a maioria dos cuidadores de familiares adoecidos, por uma questão histórica de desigualdade de gênero. 0 cuidado da família é naturalizado como responsabilidade exclusiva da mulher, ao contrário dos homens, que têm geralmente a opção de realizar o cuidado ou não, o que os leva muitas vezes a se esquivarem dessa tarefa, o que se confirmou no presente estudo. Assim, pode-se compreender o motivo de as filhas se ressentirem da falta de apoio familiar. Em sua maioria, elas são a única filha da mulher acometida pelo câncer de mama, ficando assim "naturalmente" responsáveis por todo o acompanhamento junto aos serviços de saúde, bem como pelos cuidados no âmbito doméstico, enquanto que seus pais e irmãos se esquivam ou são poupados dessas tarefas (Rodríguez \& Bertoni, 2010; Miranda \& Aravena, 2012).

Outra subcategoria consistiu no relato de três filhas que mencionaram a falta de apoio por parte dos serviços especializados como um fator dificultador do processo de lidar com a doença da mãe.

Um apoio psicológico seria ótimo. Eu senti falta, na época senti. Senti porque a cabeça da gente vira. Você perde muito o sentido. Então, acho que o hospital deveria dar também, ter essa área para as acompanhantes dos pacientes. Principalmente para a família, é o principal. Porque o paciente em si não sofre tanto quanto o familiar (Laura, 32 anos).

Esse achado é corroborado pelo estudo de Raveis e Pretter (2005), segundo o qual serviços de oncologia não dispõem de estrutura adequada para acolhimento dos familiares das muIheres com câncer de mama. As autoras afirmam que existe, entre os profissionais, sensibilidade de reconhecer a importância vital da família no decorrer do tratamento oncológico, mas que ainda assim o apoio não é oferecido sistematicamente na maioria das vezes. As falas de três filhas participantes do presente estudo tornam patente a necessidade de fornecer maior acolhimento aos familiares. Por conseguinte, é preciso considerar as demandas específicas das filhas de mulheres acometidas pelo câncer de mama. Além disso, foram mencionadas como barreiras: a necessidade de se submeter às rotinas dos serviços, as falhas de organização e a má vontade de alguns profissionais das equipes de saúde. Esses dados, vistos em seu conjunto, corroboram a necessidade de estabelecer protocolos para cuidadores de pacientes oncológicos (Moreira et al., 2012; Rezende et al., 2010).

\section{CONSIDERAÇÕES FINAIS}

Os resultados iluminaram os fatores facilitadores e dificultadores, na percepção das filhas de mulheres acometidas pelo câncer de mama, ao lidarem com o adoecimento e o tratamento oncológico de suas mães. Em relação aos aspectos facilitadores, as filhas relataram que ter fé em alguma força maior ou uma religião as auxiliou a enfrentar a doença e o tratamento da mãe. Contar com o apoio de outros familiares e com a própria força da mãe adoecida também apareceram como fatores facilitadores desse processo. Evidencia-se, assim, a importância que $o$ apoio e cuidado podem assumir na vida das filhas em um momento difícil como o acometimento da mãe por uma doença carregada de estigmas, preconceitos e má compreensão, por remeter diretamente à representação da morte no imaginário coletivo. Parece essencial, pelo que sugerem os resultados obtidos, que as filhas tenham onde buscar amparo.

Já no que concerne aos fatores que tornam o enfrentamento mais difícil por parte das filhas, os resultados mostraram que elas concordam que conviver com o estado de fragilidade da mãe é um dos aspectos mais dolorosos e que atrapalham seu processo de ajustamento, na medida em que acentuam as incertezas e temores em relação ao presente e, especialmente, ao futuro. Também relataram que a falta de apoio de familiares e de serviços médicos e oncológicos pode prejudicar o processo de enfrentamento. Diante disso, é importante ressaltar o potencial de ajuda que existe nos serviços oncológicos, uma vez que a falta desse apoio foi apontada pelas participantes como uma das principais barreiras para lidarem com a doença e tratamento da mãe. Pode-se pensar que, se os serviços oncológicos e de saúde pudessem disponibilizar às filhas de mulheres com câncer de mama maior acolhimento e oferecer respostas efetivas a algumas de suas demandas psicológicas, poderiam contribuir para fortalecer seus recursos de enfrentamento.

Os resultados coligidos contribuem para a área da saúde e dos cuidados aos pacientes oncológicos. Um ponto forte a ser destacado neste estudo é que ele abrangeu um conjunto bastante diversificado de características das filhas entrevistadas, seja em termos de faixa etária, condição socioeconômica ou estado civil. Ficou claro que as filhas são impactadas pelos eventos que afetam suas mães e que são sensíveis e permeáveis inclusive às oscilações do estado de humor da mãe adoecida, especialmente quando há falta de cooperação e aliança fraterna e privação do apoio do pai.

Este estudo pôde delinear diversos achados e conclusões, que apontam para os fatores facilitadores e dificultadores presentes no contexto de adoecimento e tratamento materno. No entanto, uma limitação que pode ser apontada nesta pesquisa é que não houve um controle em função do tempo decorrido desde o acometimento da mãe pelo câncer de mama, de forma que algumas filhas tinham passado pela experiência do 


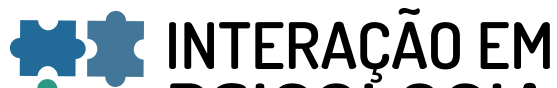 LF PSICOLOGIA}

adoecimento materno muito recentemente, enquanto outras tinham enfrentado a situação havia vários anos. Essa heterogeneidade temporal das experiências das participantes pode fazer com que fique mais difícil estabelecer padrões e generalizações a partir dos dados encontrados nesta pesquisa, ainda que caiba aqui a ressalva de que, na pesquisa qualitativa, não se almeja generalização dos achados.

Para estudos futuros, é recomendável atentar para algumas variáveis específicas, como uma pesquisa que compare a diferença entre as vivências de filhas solteiras e casadas, ou que investigue a percepção das filhas cujas mães tiveram o câncer de mama há muitos anos e outras cujas mães acabaram de completar o tratamento.

\section{FINANCIAMENTO}

Este estudo foi realizado com apoio da Fundação de Amparo à Pesquisa do Estado de São Paulo - FAPESP, processo número 2016/14831-0. O segundo autor é Bolsista de Produtividade em Pesquisa do Conselho Nacional de Desenvolvimento Científico e Tecnológico - CNPq, nível 1A.

\section{DECLARAÇÃO DA CONTRIBUIÇÃO DOS AUTORES}

Certificamos que todos os autores participaram suficientemente do estudo para tornar pública sua responsabilidade pelo conteúdo. A contribuição de cada autor pode ser atribuída como se segue:

M.G. e M.A.S. contribuíram para a conceitualização, delineamento da investigação, análise, interpretação dos resultados e revisão do artigo. M.A.S. foi responsável pela concepção e obtenção de financiamento. M.G. elaborou a redação inicial do manuscrito (rascunho) e M.G. e M.A.S. foram os responsáveis pela redação final (revisão e edição).

\section{DECLARAÇÃO DE POTENCIAIS CONFLITOS DE INTERESSE:}

Os autores declaram que não há conflitos de interesse no manuscrito submetido.

\section{REFERÊNCIAS}

Andolhe, R., Guido, L. A., \& Bianchi, E. R. F. (2009). Stress e coping no período perioperatório de câncer de mama. Revista da Escola de Enfermagem da USP, 43(3), 711-720. https:// doi.org/10.1590/S0080-62342009000300030

Ambrósio, D. C. M., \& Santos, M. A. (2011). Vivências de familiares de mulheres com câncer de mama: Uma compreensão fenomenológica. Psicologia: Teoria e Pesquisa, 27(4), 475-
484. https://doi.org/10.1590/S0102-37722011000400011

Ambrósio, D. C. M., \& Santos, M. A. (2015). Apoio social à muIher mastectomizada: um estudo de revisão. Ciência \& Saúde Coletiva, 20(3), 851-864. https://doi.org/10.1590/141381232015203.13482014

Ambrósio, D. C. M., \& Santos, M. A. (2017). Vivências de famílias de mulheres com câncer de mama: Relações e comunicações. In: E. R. Adami \& M. R. C. Chemin (Orgs.), O câncer de mama e a bioética: clínica, cuidado e prevenção (pp. 85-114). Prismas.

Araújo, J. S., \& Nascimento, M. A. A. (2004). Atuação da família frente ao processo saúde-doença de um familiar com câncer de mama. Revista Brasileira de Enfermagem, 57(3), 274278. https://doi.org/10.1590/S0034-71672004000300003

Arruda-Colli, M. N. F., Sansom-Daly, U., Santos, M. A., \& Wiener, L. (2018). Considerations for the cross-cultural adaptation of an advance care planning guide for youth with cancer. Clinical Practice in Pediatric Psychology, 6(4), 341-354. http://dx.doi.org/10.1037/cpp0000254

Benites, A. C., Neme, C. M. B., \& Santos, M. A. (2017). Significados da espiritualidade para pacientes com câncer em cuidados paliativos. Estudos de Psicologia (Campinas), 34(2), 269-279. http://dx.doi.org/10.1590/198202752017000200008

Benites, A. C., Rodin, G., Leite, A. C. A. B., Nascimento, L. C., \& Santos, M. A. (2021). The experience of spirituality in family caregivers of adult and elderly cancer patients receiving palliative care: A metasynthesis. European Journal of Cancer Care, 30(4), e13424. https://doi.org/10.1111/ecc.13424

Bergerot, C. D., Araujo, T. C. C. F., \& Tróccoli, B. T. (2014). Assessment of distress among chemotherapy patients: A comparative study of gender. Paidéia (Ribeirão Preto), 24(57), 56-65. http://dx.doi.org/10.1590/1982-43272457201408

Biffi, R. G., \& Mamede, M. V. (2010). Perception of family functioning among relatives of women who survived breast cancer: gender differences. Revista Latino-Americana de Enfermagem, 18(2), 269-277. https://doi.org/10.1590/ S0104-11692010000200019

Borges, A. D. V. S., Silva, E. F., Toniollo, P. B., Mazer, S. M., Valle, E. R. M., \& Santos, M. A. (2006). Percepção da morte pelo paciente oncológico ao longo do desenvolvimento. Psicologia em Estudo (Maringá), 11(2), 361-369. http://dx.doi. org/10.1590/S1413-73722006000200015

Brasil (2012). Resolução n 466 de 12 de outubro de 2012. Diretrizes e normas regulamentadoras de pesquisas envolvendo seres humanos. Diário Oficial da União. Brasília, DF: Conselho Nacional de Saúde. http://bvsms.saude.gov.br/ bvs/saudelegis/cns/1996/res0196_10_10_1996.html

Brasil. Instituto Nacional do Câncer José Alencar Gomes da Silva (2020). Instituto Nacional de Câncer - INCA. https:// www.inca.gov.br/tipos-de-cancer/cancer-de-mama 
Braun, V. \& Clarke, V. (2006). Using thematic analysis in psychology. Qualitative Research in Psychology, 3(2), 77-101. http://dx.doi.org/10.1191/1478088706qp063oa

Cappelli, M., Verma, S., Korneluk, Y., Hunter, A., Tomiak, E., Allanson, J. ... Humphreys, L. (2005). Psychological and genetic counseling implications for adolescent daughters of mothers with breast cancer. Clinical Genetics, 67(6), 481491. https://doi.org/10.1111/j.1399-0004.2005.00456.x

Cardoso, E. A., Mastropietro A. P., Santos M. A., \& Voltarelli, J. C. (2010). Apoio psicológico na terminalidade: Ensinamentos para a vida. Psicologia em Estudo (Maringá), 15(1), 235-244. http://dx.doi.org/10.1590/S1413-73722010000200002

Cesnik, V. M., \& Santos, M. A. (2012a). Do the physical discomforts from breast cancer treatments affect the sexuality of women who underwent mastectomy? Revista da Escola de Enfermagem da USP, 46(4), 1001-1008. https://doi. org/10.1590/S0080-62342012000400031

Cesnik, V. M., \& Santos, M. A. (2012b). Mastectomia e sexualidade: Uma revisão integrativa. Psicologia: Reflexão e Crítica, 25(2), 339-349. https://doi.org/10.1590/S010279722012000200016

Cesnik, V. M., Vieira, E. M. V., Giami, A., Almeida, A. M., Santos, D. B., Santos, M. A. (2013). The sexual life of women with breast cancer: Meanings attributed to the diagnosis and its impact on sexuality. Estudos de Psicologia (Campinas), 30(2), 187-197. https://doi.org/10.1590/S0103166X2013000200005

Compas, B. E., Worsham, N. L., Ey, S., \& Howell, D. C. (1996). When mom or dad has cancer: II. Coping, cognitive appraisals, and psycho. References logical distress in children of cancer patients. Health Psychology, 15(3), 167-175. https:// doi.org/10.1037//0278-6133.15.3.167

Davey, M., Gulish, L., Askew, J., Godette, K., \& Childs, N. (2005). Adolescents coping with mom's breast cancer: Developing family intervention programs. Journal of Marital and Family Therapy, 31(2), 247-258. https://doi.org/10.1111/j.1752-0606.2005.tb01558.x

Emslie C., Whyte F., Campbell A., Mutrie N., Lee L., Ritchie D., \& Kearney, N. (2007). "I wouldn't have been interested in just sitting round a table talking about cancer": Exploring the experiences of women with breast cancer in a group exercise trial. Health Education Research, 22(6), 827-838. https://doi. org/10.1093/her/cyl159

Ferreira, S. M. A., Gozzo, T. O., Panobianco, M. S., Santos, M. A., \& Almeida, A. M. (2015). Barriers for the inclusion of sexuality in nursing care for women with gynecological and breast cancer: Perspective of professionals. Revista Latino-Americana de Enfermagem, 23(1), 82-89. https://doi. org/10.1590/0104-1169.3602.2528

Fonseca, S., Lencastre, L., \& Guerra, M. (2014). Life satisfaction in women with breast cancer. Paidéia (Ribeirão Preto), 24(59), 295-303. https://doi.org/10.1590/198243272459201403
Freitas, I. S., Oliveira-Cardoso, E. A., \& Santos, M. A. (2017). Spirituality and religiosity in mothers of children with hematologic cancer. Psicologia em Estudo (Maringá), 22(3), 433-447. https://doi.org/10.4025/psicolestud.v22i3.34606

Hoffmann, F. S., Muller, M. C., \& Frasson, A. L. (2006). Repercussões psicossociais, apoio social e bem-estar espiritual em mulheres com câncer de mama. Psicologia, Saúde \& Doenças, 7(2), 239-254. http://www.scielo.mec.pt/scielo.php?script $=$ sci_arttext \&pid $=S 1645=00862006000200007-\&$ lngpt.

Inocenti, A., Santos, M. A., Loyola, E. A. C., Magalhães, P. A. P., \& Panobianco, M. S. (2016). Impact of the effects of the reconstructive surgery in the life of women with breast cancer. Texto \& Contexto - Enfermagem, 25(2), e4520014. http://dx.doi.org/10.1590/0104-07072016004520014

Junqueira, L. C. U., Vieira, E. M., Giami, A., \& Santos, M. A. (2013). Análise da comunicação acerca da sexualidade, estabelecida pelas enfermeiras, com pacientes no contexto assistencial do câncer de mama. Interface: Comunicação, Saúde, Educação, 17(44), 89-101. https://doi.org/10.1590/ S1414-32832013000100008

Lazarus, R. S. \& Folkman, S. (1984). Stress, appraisal and coping. Springer.

Loyola, Y. R., \& Muñíz, R. C. (2013). El diagnóstico de cáncer de mamas desde una perspectiva familiar: Retos para la Psico-oncología en América Latina. Revista Interamericana de Psicología, 47(1), 121-130. https://doi.org/10.30849/rip/ ijp.v47i1.208

Miranda, K. E., \& Aravena, V. J. (2012). Sobrecarga, apoyo social y autocuidado en cuidadores informales. Ciencia $y$ Enfermeria, 18(2), 23-30. http://dx.doi.org/10.4067/S071795532012000200003

Moreira, L. M., Ferreira, R. A., \& Costa Junior, A. L. (2012). Discussion of a care protocol for caregivers of cancer patients undergoing palliative care. Paidéia (Ribeirão Preto), 22(53), 383-392. https://doi.org/10.1590/S0103$863 \times 2012000300010$

Peres, R. S., \& Santos, M. A. (2007). Câncer de mama, pobreza e saúde mental: Resposta emocional à doença em mulheres de camadas populares. Revista Latino-Americana de Enfermagem, 15(número especial). http://www.scielo.br/pdf/ rlae/v15nspe/es_11.pdf

Peres, R. S., \& Santos, M. A. (2009). Personalidade e câncer de mama: Produção científica em psico-oncologia. Psicologia: Teoria e Pesquisa, 25(4), 611-620. https://periodicos. unb.br/index.php/revistaptp/article/view/17436

Peres, R. S., \& Santos, M. A. (2010). Elementos psicanalíticos para uma abordagem psicossomática em Psico-Oncologia. In C. M. B. Neme (Org.), Psico-Oncologia: Caminhos e perspectivas (pp. 83-98). Summus. 


\section{H MTERAC̄OAEM LF PSICOLOGIA}

Raveis, V. H., \& Pretter, S. (2005). Existential plight of adult daughters following their mother's breast cancer diagnosis. Psycho-Oncology, 14(1), 49-60. https://doi.org/10.1002/ pon. 819

Rezende, V. L., Derchain, S., Botega, N. J., Sarian, L. O., Vial, D. L., Morais, S. S., \& Perdicaris, A. A. M. (2010). Avaliação psicológica dos cuidadores de mulheres com câncer pelo General Comfort Questionnaire. Paidéia (Ribeirão Preto), 20(46), 229-237. https://doi.org/10.1590/S0103863X2010000200010

Rodríguez, S. V., \& Bertoni, J. S. (2010). Cuidado informal, um reto asumido por la mujer. Ciencia y Enfermeria, 16(2), 9-16. http://dx.doi.org/10.4067/S0717-95532010000200002

Sales, C. A., Matos, P. C. B., Mendonça, D. P. R., \& Marcon, S. S. (2010). Cuidar de um familiar com câncer: O impacto no cotidiano de vida do cuidador. Revista Eletrônica de Enfermagem, 12(4), 616-621. https://doi.org/10.5216/ree. v12i4. 12160

Sales, C. A. C. C., Paiva, L., Scandiuzzi, D., \& Anjos, A. C. Y. (2001). Qualidade de vida de mulheres tratadas de câncer de mama: Funcionamento social. Revista Brasileira de Cancerologia, 47(3), 263-272. http://www.scielo.br/pdf/csc/ v18n7/08.pdf

Sanchez, K. O. L., Ferreira, N. M. L. A., Dupas, G., \& Costa, D. B. (2010). Apoio social à família do paciente com câncer: Identificando caminhos e direções. Revista Brasileira de Enfermagem, 63(2), 290-299. https://doi.org/10.1590/S003471672010000200019

Santos, D. B., Santos, M. A., \& Vieira, E. M. (2014a). Sexualidade e câncer de mama: Uma revisão sistemática da literatura. Saúde e Sociedade, 23(4), 1342-1355. https://doi. org/10.1590/S0104-12902014000400018

Santos, D. B., Ford, N. J., Santos, M., \& Vieira, E. M. (2014b). Breast cancer and sexuality: The impacts of breast cancer treatment on the sex lives of women in Brazil. Culture, Health \& Sexuality: An International Journal for Research, Intervention and Care, 16(3), 246-257. https://doi.org/10.1080/1369105 8.2013.867075

Santos, D. B., Santos, M. A., Cesnik-Geest, V. M., \& Vieira, E. M. (2017). Interrupção e retomada da vida sexual após o câncer de mama. Psicologia: Teoria e Pesquisa, 32(4), e324219. https://dx.doi.org/10.1590/0102.3772e324219

Santos, M. A., \& Souza, C. (2019). Intervenções grupais para mulheres com câncer de mama: desafios e possibilidades. Psicologia: Teoria e Pesquisa, 35, e35410. https://doi.or$\mathrm{g} / 10.1590 / 0102.3772 \mathrm{e} 35410$

Schmid-Büchi, S., Halfens, R. J. G., Müller, M., Dassen, T., \& Borne, B. (2013). Factors associated with supportive care needs of patients under treatment for breast cancer. European Journal of Oncology Nursing, 17(1), 22-29. https://doi. org/10.1016/j.ejon.2012.02.003
Scorsolini-Comin, F., Santos, M. A., \& Souza, L. V. (2009). Vivências e discursos de mulheres mastectomizadas: Negociações e desafios do câncer de mama. Estudos de Psicologia (Natal), 14(1), 41-50. http://www.scielo.br/pdf/epsic/v14n1/ a06v14n1.pdf

Silva, B. C. A., Santos, M. A., \& Oliveira-Cardoso, E. A. (2019). Vivências de familiares de pacientes com câncer: Revisitando a literatura. Revista da SPAGESP, 20(1), 140-153. http:// pepsic.bvsalud.org/pdf/rspagesp/v20n1/v20n1a11.pdf

Silva, G., \& Santos, M. A. (2008). "Será que não vai acabar nunca?": Perscrutando o universo do pós-tratamento do câncer de mama. Texto \& Contexto - Enfermagem, 17(3), 561-568. http://www.scielo.br/pdf/tce/v17n3/a18v17n3.pdf

Silva, G., \& Santos, M. A. (2010). Stressors in breast cancer post-treatment: A qualitative approach. Revista Latino-Americana de Enfermagem, 18(4), 688-695. https://doi. org/10.1590/S0104-11692010000400005

Silva, N. C. B., Franco, M. A. P., \& Marques, S. L. (2005). Conhecimento de mulheres sobre câncer de mama e de colo do útero. Paidéia (Ribeirão Preto), 15(32), 409-416. http:// dx.doi.org/10.1590/S0103-863X2005000300010

Souza, B. F., Moraes, J. A. M., Inocenti, A., Santos, M. A., Silva, A. E. B. C., \& Miasso, A. I. (2014). Women with breast cancer taking chemotherapy: Depression symptoms and treatment adherence. Revista Latino-Americana de Enfermagem, 22(5), 866-873. https://doi.org/10.1590/01041169.3564.2491

Tavares, J. S. C., \& Trad, L. A. B. (2005). Metáforas e significados do câncer de mama na perspectiva de cinco famílias afetadas. Cadernos de Saúde Pública, 21(2), 426-435. https://doi.org/10.1590/S0102-311X2005000200009

Tonon, L., Peres, R. S., \& Santos, M. A. (2016). Crescimento pós-traumático e relações interpessoais em mulheres acometidas por câncer de mama. In D. Bartholomeu, J. M. Montiel, A. A. Machado, A. R. Gomes, G. Couto, \& V. Cassep-Borges (Orgs.), Relações interpessoais: concepções e contextos de intervenção e avaliação (pp. 299-312). Vetor.

Vidotti, J. F., Scorsolini-Comin, F., \& Santos, M. A. (2013). Qualidade de vida em sobreviventes de longo prazo ao câncer de mama: Análise da produção científica. Psicologia: Teoria e Prática, 15(3), 49-68. http://pepsic.bvsalud.org/pdf/ptp/ v15n3/04.pdf

Vieira, E. M., Santos, D. B., Santos, M. A., \& Giami, A. (2014). Experience of sexuality after breast cancer: A qualitative study with women in rehabilitation. Revista Latino-Americana de Enfermagem, 22(3), 408-414. https://doi. org/10.1590/0104-1169.3258.2431

Vieira, E. M., Ford, N. J., Santos, M. A., Junqueira, L. C. U., \& Gia$\mathrm{mi}, \mathrm{A}$. (2013). Representations of nurses regarding sexuality of women treated for breast cancer in Brazil. Cadernos de Saúde Pública, 29(10), 2049-2056. https://doi.org/10.1590/ 0102-311X00158512 


\section{MI" INTERACÃO EM \\ ET PSICOLOGIA}

Vodermaier, A., \& Stanton, A. L. (2012). Familial breast cancer: less emotional distress in adult daughters if they provide emotional support to their affected mother. Familial Cancer, 11(4), 645-652. https://doi.org/10.1007/s10689-0129566-y

Walks, A. G., \& Winer, E. P. (2019). Breast cancer treatment: A review. JAMA, 321(3), 288-300. https://doi.org/10.1001/ jama.2018.19323

Yates, P. (1999). Family coping: Issues and challenges for cancer nursing. Cancer Nursing, 22(1), 63-71. https://doi. org/10.1097/00002820-199902000-00012
Yoshimochi, L. T. B., Santos, M. A., Loyola, E. A. C., Magalhães, P. A. P., \& Panobianco, M. S. (2018). The experience of the partners of women with breast cancer. Revista da Escola de Enfermagem da USP, 52, e03366. https://doi.org/10.1590/ s1980-220x2017025203366

Data de submissão: 17/04/2020

Primeira decisão editorial: 29/10/2020

Aceite em 03/12/2020 